\title{
Estrategias para la elección de carrera y rutas de ingreso a la universidad*
}

\section{Ignacio Pérez Pulido}

https://orcid.org/0000-0002-8215-8850 Universidad de Guadalajara, México iperez@cualtos.udg.mx

\section{Luis Felipe Gómez López}

https://orcid.org/0000-0001-6988-0163 Instituto Tecnológico y de Estudios Superiores de Occidente, México lgomez@iteso.mx

\section{Resumen}

Este artículo busca identificar las estrategias utilizadas por un grupo de estudiantes durante su proceso de ingreso a una carrera profesional en una universidad oficial mexicana. El estudio se realizó desde el enfoque cualitativo, mediante doce entrevistas en profundidad con estudiantes de las carreras de Medicina, Enfermería e Ingeniería en Sistemas Pecuarios. La información se interpretó mediante las categorías de habitus, campo y capital de Pierre Bourdieu. El análisis identificó cómo surge el interés por entrar a una carrera universitaria, asi como cuatro rutas de ingreso: elección temprana, valoración de posibilidades, elección tardia poco informada y cambio de aspiración, y la variante: volver a hacer trámites. Como conclusión, se muestra que el ingreso ocurre en un proceso dialéctico entre las subjetividades de los estudiantes (capital $y$ habitus) y las reglas y condiciones que establece el campo universitario.

\section{Palabras clave (Fuente: tesauro Unesco)}

Acceso a la educación; asesoramiento; acceso a la educación universitaria; elección profesional; educación superior; orientación profesional.

\footnotetext{
* $\quad$ Artículo derivado de la tesis de doctorado "El proceso de adaptación de los estudiantes a la universidad en el Centro Universitario de Los Altos de la Universidad de Guadalajara”. Doctorado Interinstitucional en Educación DIEE, Instituto Tecnológico y de Estudios Superiores de Occidente (ITESO), Guadalajara, Jalisco.
} 


\title{
Career Choice Strategies and Pathways to College*
}

\begin{abstract}
The research seeks to identify a group of students'strategies for joining an undergraduate program at a Mexican public university. We carried it out from a qualitative approach through twelve in-depth interviews with students of Medicine, Nursing, and Livestock Systems Engineering. The information was interpreted using Pierre Bourdieu's habitus, field, and capital categories. The analysis identified how the interest in enrolling in an undergraduate program arises, four pathways to college (early choice, assessment of possibilities, poorly informed late choice, and change of aspiration), and a variant (doing paperwork again). Therefore, going to college results from a dialectical process between the students' subjectivities (capital and habitus) and the university's rules and conditions.
\end{abstract}

\section{Keywords (Source: Unesco Thesaurus)}

Access to education; access to higher education; occupational choice; higher education; career guidance; counselling.

* $\quad$ Article derived from the doctoral dissertation "El proceso de adaptación de los estudiantes a la universidad en el Centro Universitario de Los Altos de la Universidad de Guadalajara." Interinstitutional Doctorate in Education (DIEE), Instituto Tecnológico y de Estudios Superiores de Occidente (ITESO), Guadalajara, Jalisco. 


\section{Estratégias para escolher a profissão e caminhos para entrar na universidade*}

\section{Resumo}

Nesta pesquisa, procura-se identificar as estratégias utilizadas por um grupo de estudantes durante seu processo de ingresso a um curso superior oficial em uma universidade mexicana. O estudo foi realizado sob a abordagem qualitativa, mediante 12 entrevistas em profundidade com estudantes de Medicina, Enfermagem e Engenharia de Sistemas Pecuários. Os dados foram interpretados com base nas categorias de habitus, campo e capital de Pierre Bourdieu. Na análise, foi identificado como o interesse por fazer um curso superior surge, bem como quatro caminhos de ingresso: escolha precoce, avaliação de possibilidades, escolha tardia pouco informada e mudança de aspiração, além da variante "voltar a realizar processos". Como conclusão, mostra-se que o ingresso ocorre em um processo dialético entre as subjetividades dos estudantes (capital e habitus) e as regras e as condições que o campo universitário estabelece.

\section{Palavras-chave (Fonte: tesauro da Unesco)}

Acesso à educação; conselho; acesso à educação universitária; escolha profissional; ensino superior; orientação profissional.

Artigo derivado da tese de doutorado "El proceso de adaptación de los estudiantes a la universidad en el Centro Universitario de Los Altos de la Universidad de Guadalajara" “El proceso de adaptación de los estudiantes a la universidad en el Centro Universitario de Los Altos de la Universidad de Guadalajara". Doctorado Interinstitucional en Educación DIEE, Instituto Tecnológico y de Estudios Superiores de Occidente (ITESO), Guadalajara, Jalisco. 
Ingresar a la universidad puede ser visto por los estudiantes y sus personas cercanas como una gran oportunidad que en el futuro puede traer beneficios profesionales y laborales. Por otro lado, aunque no es evidente, existen estructuras sociales que facilitan o dificultan el ingreso y la permanencia en la universidad. De esta manera, los jóvenes de familias de altos estratos sociales tienen más posibilidades de continuar sus estudios superiores en comparación con aquellos que provienen de familias de estratos económicos bajos (Bourdieu y Passeron, 2003; Grodsky y Riegle, 2010); sin embargo, algunos de estos despliegan estrategias que les permiten ingresar y permanecer en la universidad, lo que en muchas ocasiones es percibido como un logro y una oportunidad de mejorar sus condiciones laborales (Guerra, 2009) y, en general, de ascenso social. Lo anterior implica reconocer a los estudiantes universitarios como un grupo heterogéneo y diverso (De Garay, 2004; Miller, 2015) y entenderlos como sujetos con experiencias y voz propias, insertos en contextos institucionales específicos (Guzmán y Saucedo, 2005). De esta manera, el ingreso a la universidad puede entenderse como un proceso en el que intervienen circunstancias personales, sociales, académicas e institucionales que facilitan u obstaculizan la trayectoria de los estudiantes (Hegna, 2014).

Las investigaciones que han abordado el proceso de ingreso a la universidad se han enfocado en distintos factores que permiten identificar los elementos que favorecen o no el acceso de los estudiantes a ella; entre otros, destacan: las características socioculturales del estudiante y su familia (Bernardi y Cebolla, 2014; De Garay, 2004; González, 2014; Silva y Rodríguez, 2012); las características de la escuela de procedencia (González, 2016; Langenkamp y Shifrer, 2011; Reay et al., 2001); la trayectoria escolar precedente (Bernardi y Cebolla, 2014; Rodríguez et al., 2004); las características de la institución universitaria (González, 2016); los efectos de la orientación vocacional (Subero y Esteban, 2017); y la influencia del género en la elección de la carrera (Rodríguez et al.,
2016), cuando la carrera cursada fue la primera opción (Rodríguez et al., 2004).

Lo anterior hace evidente que el ingreso a la universidad y, en específico, la elección de carrea constituyen un proceso sumamente complejo para los estudiantes, que se ha estudiado desde diferentes perspectivas, entre las que destaca la teoría de la acción racional. Esta plantea que durante la transición a la educación superior los estudiantes valoran oportunidades y limitaciones a partir de sus características personales, familiares, académicas y sociales, lo que puede ocasionar que tomen diversas trayectorias y que -a partir de los consejos de sus familiares, de sus propias creencias respecto al costo académico, riesgo de rechazo y retorno económico de las carreras e instituciones- tomen la decisión (Piñero, 2015).

Otro enfoque desde el que se ha abordado la elección de carrera es la perspectiva de género, que ha permitido identificar que, a pesar de que en los últimos años se ha incrementado el ingreso de las mujeres a la educación superior (Macías et al., 2011), aún existen fuertes diferencias respecto al tipo de carreras que eligen hombres y mujeres, las cuales se explican a partir de los estereotipos de género, de manera que ciertas carreras son consideradas como femeninas o masculinas (Navarro y Casero, 2012). Esto puede limitar la elección, viéndose especialmente afectadas las mujeres, para quienes estarían reservadas las profesiones relacionadas con los cuidados, la imagen y la limpieza, mientras que para los hombres estarían reservadas aquellas que requieren fuerza física (García et al., 2015).

Los estudios destacan que el sistema de educación superior en México es altamente heterogéneo, compuesto por universidades tanto públicas como privadas, todas ellas con sistemas de admisión diferentes. Las primeras se caracterizan por bajas o nulas colegiaturas y una demanda de aspirantes que sobrepasa sus capacidades de admisión, mientras que en las privadas el factor económico es uno de los 
principales criterios de ingreso y, a la vez, de calidad. Lo anterior ha promovido investigaciones enfocadas en identificar los factores socioeconómicos y culturales que influyen en el ingreso a la universidad (Pérez y Villarruel, 2016; De Garay, 2004) y otras que analizan las acciones de los aspirantes que no son admitidos en las universidades públicas (Guzmán, 2012).

Una de estas universidades públicas es el Centro Universitario de Los Altos (cualtos), un campus regional de la Universidad de Guadalajara (UdeG), cuyo propósito principal es brindar educación superior a los habitantes de la región de Los Altos, al sur de Jalisco. Es un campus multitemático que ofrece 13 programas de licenciatura en las áreas agropecuarias, de la salud, económico-administrativas, de computación y de derecho. El mecanismo de admisión establecido por la UdeG parte de una ponderación a partes iguales del promedio de bachillerato y el puntaje obtenido en el examen de aptitud académica College Board. Con ello, la Universidad define los méritos académicos de los aspirantes como parámetro de ingreso (Ochoa, 2000).

La presente investigación, que se llevó a cabo en el cualtos, tuvo como objetivo caracterizar las estrategias y recursos desplegados por los aspirantes para ingresar a la universidad. Para ello, se apoya en los conceptos teóricos de campo, capital y habitus desarrollados por Pierre Bourdieu y reconoce a los estudiantes como sujetos activos, capaces de tomar sus propias decisiones. El estudio se apoya en la sociología estructural constructivista, desarrollada por Bourdieu para hacer evidentes las acciones prácticas desplegadas por los estudiantes, encaminadas a lograr el ingreso y la permanencia en la universidad. Para ello, se retoman los conceptos de habitus, capital y campo, como herramientas teóricas que, de manera complementaria, permiten demostrar que cada sujeto cuenta con condiciones diferentes que facilitan o dificultan su ingreso a la universidad; a su vez, esta última se entiende como una estructura social que impone reglas a las que el aspirante debe responder.
En primer término, se entiende el habitus como "un sistema socialmente constituido de disposiciones estructuradas y estructurantes adquirido mediante la práctica y siempre orientado hacia funciones prácticas" (Bourdieu y Wacquant, 1995, p. 83). De aquí se desprende que los sujetos adquieren de las estructuras sociales una serie de disposiciones que los inclinan a actuar de un modo determinado, si bien al sujeto se le reconoce la capacidad de actuar de manera distinta. El habitus permite enlazar el mundo objetivo de las estructuras sociales (el campo) con el mundo subjetivo de los agentes (Farías, 2010). Es a través de este concepto que Bourdieu reconoce en el sujeto la capacidad de actuar y tomar decisiones propias, aunque no totalmente libres de las disposiciones establecidas por las estructuras sociales. "La función principal de la noción de habitus está en la superación de una visión dualista que separa indebidamente al agente de la estructura" (Velasco, 2000, p. 35).

Para comprender con más detalle cómo funciona el habitus, es pertinente destacar la relación dialéctica de sus dos componentes: la hexis y la illusio. La primera se puede entender como un sistema duradero de disposiciones aprendidas socialmente que predisponen a actuar, mientras que la segunda representa un interés, una creencia por parte del individuo, que le permite generar expectativas dentro de un campo (Mota, 2013). De esta manera, no se debe entender el habitus como un sistema determinista que predispone a actuar, sino como un "resorte que precisa de un disparador: [pues] dependiendo de los estímulos y de la estructura del campo, el mismo habitus generará resultados distintos, incluso opuestos" (Bourdieu y Wacquant, 1995, p. 198).

Investigaciones recientes se han apoyado en este concepto para identificar las disposiciones incorporadas que impulsan a los estudiantes a ingresar a la universidad. En algunas, se lo ha definido como habitus institucional (González, 2016; Langenkamp y Shifrer, 2011), que se entiende como las disposiciones incorporadas a través de la socia- 
lización en la escuela, específicamente en el nivel de estudios previo, y que impulsan a los estudiantes a ingresar a la universidad. Otras investigaciones plantean el habitus de ingreso a la universidad (Grodsky y Riegle, 2010; Langenkamp y Shifrer, 2011), al que describen como las disposiciones que se incorporan a través de la socialización del estudiante, misma que ubican principalmente en la familia, aunque no se restringe a esta, y que le permiten desde edades tempranas dar por sentado que ingresará a la universidad.

Considerando que las disposiciones que impulsan a ingresar a la universidad pueden ser incorporadas en distintos espacios sociales, ya sean familiares, escolares u otros, y que para ingresar a la universidad deben existir disposiciones y expectativas tanto escolares como profesionales, pues ambas son requeridas e inculcadas en el campo universitario, el presente estudio utilizará el término habitus de ingreso a la universidad, con el fin de identificar cómo surge el interés por estudiar una carrera universitaria y relacionarlo con los distintos tipos de capital y el campo.

Otro de los conceptos clave desarrollados por Bourdieu es el de capital, donde destacan tres clases fundamentales: el económico, el cultural y el social (Bourdieu y Wacquant, 1995). Contar con determinados capitales permite a los agentes posicionarse dentro del campo, de manera que poseer ciertos grados y tipos de capital brinda beneficios específicos $y$, de igual manera, carecer de ellos deja al aspirante en desventaja (Bourdieu, 1990).

Por último, está el concepto de campo, que se entiende como un espacio social estructurado, compuesto por agentes con intereses en común, que detentan diferentes grados de autoridad y compiten por los recursos o capitales afines. El funcionamiento del campo requiere que existan capitales en juego y agentes dotados de un habitus que les permita conocer y comprender las reglas del juego y los impulse a valorar y competir por dichos capitales
(Bourdieu, 2002). El principal capital que se disputa en el campo universitario es el cultural (Bourdieu, 2008), pues brinda a quienes participan un tipo de reconocimiento que posteriormente puede traducirse en diferentes beneficios, en especial, en capital económico y simbólico.

En general, los planteamientos de Bourdieu han permitido el desarrollo de diversas investigaciones que ponen el acento en la reproducción de las desigualdades y sus consecuencias en la educación superior; sin embargo, este conjunto de herramientas tiene el potencial de develar los mecanismos de esta reproducción (Edgerton y Roberts, 2014). Entendido de esta manera, el instrumental teórico desarrollado por Bourdieu permite reconocer al estudiante como un actor capaz de establecer sus propias estrategias de ingreso impulsadas por su habitus y sustentadas en sus capitales y, a la vez, identificar y comprender las valoraciones y acciones prácticas del estudiante encaminadas a responder a las reglas del campo universitario, entendido como estructura social.

\section{Método}

La presente investigación es de tipo cualitativo, ya que interesaba analizar casos específicos que permitieran profundizar en la perspectiva de los estudiantes, sus prácticas y disposiciones en torno a su proceso de ingreso a la universidad, pues existen variables que no se pueden aprehender a través de datos estadísticos y requieren ser expresadas y en ocasiones explicadas por el propio estudiante a través de la verbalización de sus experiencias. Debido a lo anterior, se estimó que la entrevista es un instrumento útil en este tipo de condiciones, ya que permite obtener información sobre acontecimientos y actividades que no se pueden observar directamente, ya sea porque sucedieron en el pasado o porque el investigador no tiene acceso a ellas (Taylor y Bogdan, 1996); además, permite percibir las interpretaciones y los significados que los estudiantes dan a sus experiencias (Warren, 2001) durante su proceso de ingreso. 
En total, se realizaron 24 entrevistas a doce estudiantes de primer semestre durante el año 2014. La selección de los participantes se apoyó en un estudio cuantitativo realizado previamente que permitió identificar características de interés tanto de los estudiantes como de las carreras, a las cuales se las puede entender como subcampos dentro del Centro Universitario de Los Altos (cualtos). Uno de los criterios de selección se enfocó en las características de las carreras y se consideraron dos variables: a) el puntaje mínimo de ingreso a cada carrera, el cual constituye un parámetro que permite identificar los capitales culturales y el habitus de ingreso a la universidad de los estudiantes, así como su efecto conjunto en la carrera. Visto desde la colectividad, se plantea que a mayor puntaje de ingreso se esperarían mayores capitales culturales y un habitus más afín a la universidad; y b) el porcentaje de estudiantes que ingresaron a la carrera como primera opción, parámetro que permitió detectar carreras con altos porcentajes de estudiantes que cambiaron su aspiración; en este sentido, la investigación buscó comprender las razones del cambio y qué hacen estos jóvenes para ajustar su aspiración a una nueva carrera.

Atendiendo al criterio anterior, se seleccionaron doce estudiantes que pertenecieran a las carreras de: a) Medicina, por registrar los mayores puntajes de ingreso y a la vez contar con el menor porcentaje de estudiantes que ingresaron a una carrera distinta a la que deseaban estudiar; b) Ingeniería en Sistemas Pecuarios, que mostró los puntajes de ingreso más bajos y el mayor porcentaje de estudiantes que ingresaron a una segunda opción; y c) Enfermería, que registró un alto porcentaje de estudiantes que deseaban ingresar a otra carrera y un puntaje de ingreso intermedio.

Una vez definidas las carreras, se seleccionaron cuatro estudiantes de cada una, con los siguientes criterios de inclusión: a) puntaje de ingreso a la universidad, entendido como un indicador que permite identificar la incorporación de disposiciones afines a la universidad; b) escolaridad de los padres, para detectar capitales culturales; y c) haber ingresado a la carrera deseada o a una distinta, lo que permitió diferenciar las estrategias de ingreso. Se buscó equilibrar dichos atributos entre los participantes, de manera que al momento de realizar el análisis fuera factible establecer comparaciones. En la Tabla 1 se muestran las características de cada participante.

Tabla 1. Caracteristicas de los participantes

\begin{tabular}{|c|c|c|c|}
\hline Caracteristicas & & 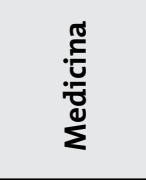 & 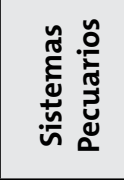 \\
\hline $\begin{array}{l}\text { - Alto puntaje de ingreso } \\
\text { - Padres con capitales } \\
\text { culturales bajos } \\
\text { - Primera opción }\end{array}$ & Raquel & Susana & José \\
\hline $\begin{array}{l}\text { - Alto puntaje de ingreso } \\
\text { - Padres con capitales } \\
\text { culturales altos } \\
\text { - Segunda opción }\end{array}$ & Pedro & Salvador & Gerardo \\
\hline $\begin{array}{l}\text { - Bajo puntaje de ingreso } \\
\text { - Padres con capitales } \\
\text { bajos } \\
\text { - Primera opción }\end{array}$ & Adelita & Mary & Gonzalo \\
\hline $\begin{array}{l}\text { - Bajo puntaje de ingreso } \\
\text { - Padres con capitales } \\
\text { culturales altos } \\
\text { - Segunda opción }\end{array}$ & Betty & Andrea* & Luis \\
\hline
\end{tabular}

Fuente: elaboración propia.

La selección de participantes con atributos similares e inscritos en carreras que presentan distintos contextos permitió establecer comparaciones y detectar diferencias, a partir de la subjetividad de cada estudiante y de sus características individuales. Además, permitió identificar cómo influye el contexto de cada carrera en el proceso de ingreso a la universidad.

El análisis de los datos se basó en un proceso inductivo, donde la codificación surgió de manera empírica y permitió construir explicaciones del fenó- 
meno de ingreso a la universidad que incluyen cuatro rutas más una variante. Los datos se codificaron y categorizaron; la información obtenida se desplegó en tablas y matrices de doble entrada, y, mediante un proceso inductivo, se extrajeron las conclusiones.

\section{Papel del habitus en el ingreso a la universidad}

El concepto de habitus y sus componentes hexis e illusio permitieron explicar cómo surgió el interés por la carrera y las acciones prácticas desplegadas por los estudiantes para lograr el ingreso. Cabe señalar que, desde que surgió la expectativa de ingresar a la universidad, esta se fue consolidando a través de las acciones del estudiante, que a su vez fortalecieron la expectativa. En otras palabras, primero surgió la illusio que consolida la hexis, que a su vez modifica y refuerza la illusio en una dinámica recurrente.

\section{Génesis del habitus: los modelos como precursores de la illusio}

Los datos analizados permiten identificar el interés por una carrera universitaria como el germen de la illusio y, por tanto, del habitus de ingreso a la universidad, pues es a partir de este interés que el estudiante empieza a generar expectativas e incorporar disposiciones que lo impulsan a emprender acciones prácticas encaminadas a ingresar al campo universitario. Considerando que la mayoría de los estudiantes del cualtos proviene de contextos familiares alejados de la universidad, se hace necesario identificar cómo se genera el interés por cursar estudios superiores.

Nueve estudiantes tuvieron algún tipo de contacto con profesionistas de la carrera que estudian, a partir del cual se generó un interés. Es importante destacar que en la mayoría de los casos este contacto tuvo un carácter casual, por lo que el profesionista que fue tomado como modelo no tuvo ninguna acción intencionada. Como relata Adelita, "cuando estaba en la secundaria conocí a una vecina que era enfermera y me gustó que atendía a su hijo y traba- jaba. No sabía qué hacía, pero me gustó". Incluso en dos de estos casos el modelo fue un personaje ficticio, como el protagonista de una serie de televisión o una película. Lo importante de estas interacciones es que fueron capaces de inculcar expectativas respecto a estudiar una carrera profesional y en algunos estudiantes provocaron la incorporación gradual de disposiciones afines a la carrera. Estas interacciones hacen evidente que el interés por estudiar una carrera universitaria puede surgir de contactos con profesionistas fuera del seno familiar.

De los estudiantes entrevistados, solo tres no mencionaron un modelo específico. Esto se explica por lo siguiente: Salvador cambió de carrera y su illusio estaba en Computación, pero cuando tomó la decisión de ingresar a Medicina le gustó el prestigio de la carrera y que no cualquiera entraba; la vio como un reto, y se puede decir que el interés por la carrera vino de las características de la misma. Con Pedro sucedió algo similar: al cambiar de carrera, su interés se ubicó en las características de la carrera a la que ingresó. El caso de Gerardo es distinto, pues constantemente mencionó que quería ser policía y su illusio tendía más a dicha profesión que a Sistemas Pecuarios; su modelo no era compatible con la carrera. En este caso, el ingreso se explica debido a que su mamá se negó a que fuera policía.

Es difícil separar las expectativas de las disposiciones o la illusio de la hexis, pues, como ya se mencionó, una fortalece a la otra. Para ilustrar cómo influye el modelo en la generación de expectativas y en la incorporación de disposiciones, se toma el ejemplo de Gonzalo, quien durante la secundaria tuvo un profesor agrónomo de profesión y dueño de un invernadero. A Gonzalo le gustó que su profesor cultivara frutas y verduras, independientemente de la temporada que fuera, y comenzó a ayudarle esporádicamente. Estas pequeñas interacciones a la postre tuvieron la capacidad de generar una illusio en Gonzalo, que lo llevó a ingresar a la carrera de Sistemas Pecuarios, pero al mismo tiempo adquirió conocimientos y disposiciones afines a esta actividad profesional. 
En su inicio, el interés por ingresar a una carrea puede tener un carácter vago y difuso y manifestarse como un gusto por un área de conocimiento y no tanto por una carrera en específico. Este tipo de manifestaciones permite ver que los aspirantes tienen nociones de lo que es una carrera universitaria, pero no necesariamente la conocen a fondo; sin embargo, el interés por la carrera cobra importancia en la medida en que es el impulsor para que el aspirante emprenda acciones para ingresar a la misma.

El interés por la carrera puede aparecer a edades muy tempranas. Ocho de los entrevistados manifestaron que su gusto por una carrera surgió durante la secundaria o antes, tres expresaron que surgió durante la preparatoria y uno no mencionó un momento en específico. Lo anterior se puede tomar como una evidencia de la complejidad del proceso de ingreso a la universidad y de que puede iniciar mucho tiempo antes de ingresar al campo universitario, cuando aparece la illusio, entendida como el interés y la expectativa de estudiar una carrera.

\section{Génesis de la hexis: la incorporación de disposiciones escolares y profesionales}

Para el caso particular del habitus de ingreso a la universidad se pueden identificar dos tipos de disposiciones que conforman la hexis: las escolares y las profesionales. Las primeras son las que cualquier campo escolar demanda, por ejemplo, la docilidad, que se entiende como la disposición a dejarse enseñar, la elaboración de tareas, el respeto al profesor, etc. Mientras que las disposiciones profesionales son aquellas que demanda y valora el campo profesional al que el estudiante pretende ingresar, una vez terminados sus estudios universitarios, por ejemplo: comprender las labores del rancho, en el caso de un estudiante de Sistemas Pecuarios, o estar familiarizado con las reglas y funcionamiento de un hospital, para los estudiantes de Medicina y de Enfermería.

Los datos analizados permitieron identificar que los estudiantes presentaron interacciones más o menos constantes con agentes específicos que fo- mentaron la incorporación de ambas disposiciones y que existen diferencias en el grado de incorporación y afinidad con el campo universitario. De manera similar a la illusio, unas y otras surgieron y se fortalecieron antes del ingreso a la universidad en momentos y circunstancias diferentes. A continuación, se describen las interacciones sociales de los estudiantes que les permitieron incorporar disposiciones que desembocaron en el ingreso al campo universitario.

El escenario privilegiado para la incorporación de las disposiciones escolares es el campo escolar, entendido como los niveles de estudio previos por los que transitaron los estudiantes; en otras palabras, el campo donde naturalmente se incorporan las disposiciones escolares es la escuela en sus diferentes niveles. El haber obtenido el certificado de preparatoria es una prueba de su tránsito por campos escolares donde adquirieron ciertas disposiciones, mientras que el promedio de la preparatoria es un indicador de qué tan sólidas son estas disposiciones y de su afinidad con el campo universitario.

Nueve de los estudiantes presentaron promedios superiores al 85, lo que indica que tuvieron un buen desempeño en la preparatoria, por lo que se puede considerar que cuentan con disposiciones afines o muy afines a las demandadas por el campo escolar, mientras que tres estudiantes obtuvieron promedios aproximados de 75, con un resultado entre satisfactorio y bajo; sin embargo, como estos promedios son solo un registro numérico, únicamente brindan un referente de las disposiciones escolares y no las explican, lo que hace necesario el análisis de datos cualitativos para identificar cómo son y cómo se construyeron las disposiciones escolares de los alumnos entrevistados.

Cinco estudiantes manifestaron comportamientos muy afines a los valores del campo escolar: Susana, Andrea, Raquel, Salvador y Pedro mencionaron que, a pesar de que no realizaban muchos esfuerzos, conseguían buenas calificaciones. Esto se 
evidencia en sus comentarios. Así, cuando Susana dice: "en la preparatoria siempre me iba bien en mis exámenes, estaba acostumbrada a eso", la aparente no necesidad de esfuerzo muestra que sus disposiciones concordaban con las requeridas por el campo. Por su lado, Adelita manifiesta que desde la preparatoria le gustaba esforzarse por cumplir con sus tareas y atender a las peticiones de sus maestros: "me daba vergüenza que mis profesores dijeran que hacía mal las cosas", lo que muestra que ella consideraba valioso e importante cumplir con las reglas de la escuela. Esto la llevó a incorporar disposiciones de estudio y elaboración de tareas, que le permitieron responder adecuadamente a las reglas del campo.

Destaca el caso de Mary, quien tuvo la capacidad de modificar su comportamiento en la preparatoria con el fin de responder mejor a las reglas del campo escolar y elevar sus calificaciones. Para lograrlo incorporó disposiciones como: estudiar, entregar tareas, etc. Estos cambios fueron motivados porque un profesor le advirtió que con calificaciones bajas no entraría a Medicina. La expectativa reconfigurada -que incluía la posibilidad de no ingresar a la carrera deseada y de que, para lograrlo, requería mejores calificaciones- la impulsó a incorporar disposiciones escolares más sólidas. Los ejemplos mostrados permiten identificar que los estudiantes incorporaron disposiciones escolares de manera previa a su ingreso a la universidad y confirman que estas pueden facilitar o dificultar el ingreso y la permanencia en ella.

El segundo componente de la hexis son las disposiciones profesionales. La identificación y análisis del origen y desarrollo de estas se enfrentó a la dificultad de que generalmente los aspirantes no se ubicaban en estructuras sociales o campos capaces de promover el contacto con agentes sociales que faciliten la incorporación de disposiciones profesionales, como lo es la escuela para las disposiciones escolares.

La mayoría de los estudiantes manifestaron pocas acciones que los llevaran a adentrarse al cam- po profesional de su carrera, incluso en algunos no se generaron y la mayoría de estas acciones fueron incipientes acercamientos al campo profesional. Por ejemplo, las pláticas de Mary con médicos para conocer más la profesión, las cuales fomentaron el gusto por la carrera de Medicina, aunque no realizó ninguna acción práctica. Esta situación es más evidente en el caso de los acercamientos en situaciones irreales, como el ver series de televisión, en el caso de Susana, en las cuales, a pesar de estar fundamentadas en situaciones y personajes ficticios, fueron capaces de incorporar en ella incipientes disposiciones afines a la profesión. Esta limitada incorporación de disposiciones profesionales se debe a que los estudiantes en realidad no están en el campo profesional, por lo que un acercamiento es poco probable, a menos que socialicen con un profesionista del campo y los adentre al mismo.

Por lo que toca a los estudiantes de Sistemas Pecuarios, destaca que, gracias a la vocación agropecuaria de las localidades en donde residen, tuvieron contactos directos con el campo profesional de manera más o menos cotidiana y constante, sobre todo a nivel práctico. Por ejemplo, José acompañaba a su padre al rancho, donde se adentró de manera empírica en el campo agropecuario; sin embargo, no se registraron contactos específicos con ingenieros en Sistemas Pecuarios que le permitieran comprender mejor el campo profesional de esta carrera, sino hasta que él mismo buscó a egresados de la misma para conocerla.

En resumen, la hexis del habitus de ingreso a la universidad se construye a través de la consolidación gradual de dos disposiciones: por un lado, las escolares, que permiten actuar de manera satisfactoria en la escuela y, por otro, las profesionales, que se generan en el contacto con agentes capaces de promover prácticas cercanas a la profesión deseada. Valga señalar que sería muy raro generar este tipo de contactos fuera del campo profesional; por ello, antes de entrar a la universidad es común que la hexis se presente poco consolidada, pero deberá 
ser lo suficientemente fuerte como para permitir la entrada al campo universitario, el cual se entiende como la estructura social que facilita y promueve la incorporación de las disposiciones profesionales, a través de prácticas escolares específicas.

\section{Las rutas de ingreso a la universidad}

La elección de carrera es un momento clave del proceso de ingreso a la universidad, pues es donde confluyen la subjetividad del aspirante y las reglas del campo universitario. Es cuando el aspirante, guiado por su illusio e impulsado por su hexis, pone en juego sus capitales cultural, económico y social con el fin de ingresar al campo universitario. Este momento se puede describir como una acción práctica en la que el aspirante valora sus posibilidades de ingresar y elige una carrera respecto de la cual hacer trámites, misma que puede ser su carrera de interés o una distinta.

Durante la elección de carrera el estudiante se enfrenta al campo universitario, entendido como estructura social que ofrece capitales y establece reglas, en este caso, para la admisión. En este momento es cuando pueden realizar ajustes que le permitan responder adecuadamente a ellas. En la medida en que el estudiante comprenda las reglas estará en mejores condiciones para interactuar conscientemente con el campo universitario. Atendiendo a esto, el análisis de los datos resaltará los momentos en los que los estudiantes percibieron estas reglas y emprendieron acciones específicas para responder a ellas.

Si bien cada estudiante generó un proceso de elección distinto, se identificaron ciertas coincidencias a partir de las cuales se generaron cuatro rutas de ingreso: 1) elección temprana, 2) elección temprana con valoración de posibilidades, 3) elección tardía y poco informada y 4) cambio de aspiración. Estas elecciones pudieron presentar la variante de volver a hacer trámites. A continuación, se describe cada ruta de ingreso a partir del proceso de elección.

\section{Ruta 1: elección temprana}

La elección temprana está muy relacionada con el interés por la carrera y, por lo mismo, es difícil diferenciar entre interés y elección. Dos estudiantes siguieron esta ruta: Adelita de Enfermería y Mary de Medicina. Ellas se distinguen por haber mostrado un interés por la carrera desde la secundaria y porque sus acciones las llevaron a incorporar de manera gradual disposiciones y a generar expectativas que consolidaron su gusto por la carrera, lo que a la par les ayudó a adquirir capitales sociales y culturales que les permitieron ingresar al campo universitario. A estos momentos se les llamó reforzamientos del interés y en conjunto desembocaron en el ingreso de la carrera deseada. En estas estudiantes se detectaron acciones encaminadas a conocer aspectos concretos de la carrera que deseaban estudiar y cambios de conducta e incorporación de ciertas disposiciones con el fin específico de ingresar a la carrera. A continuación se expone el caso de Adelita.

Adelita empezó a interesarse en Enfermería cuando estudiaba la secundaria a partir de contactos casuales con una enfermera, a quien tomó como modelo. Se aprecia que al principio no comprendía en qué consistía la carrera, pero estos contactos fueron lo suficientemente potentes como para generar una illusio con el referente de las enfermeras, como personas responsables y esforzadas que mostraban logros asociados a su actividad y deseó de llegar a hacer la misma actividad que el modelo. Lo refirió así: "ella se iba a trabajar y tenía un hijo estudiando y me gustó eso". Esta illusio se fue fortaleciendo con otro tipo de acciones como ver películas relacionadas con la enfermería.

Durante la preparatoria Adelita cursó la materia Taller de Educación para la Salud donde adquirió conocimientos relacionados con la carrera de su interés; además, su profesor le permitió realizar algunas acciones concretas que fueron confirmando su gusto por la carrera: "el Doctor nos invitó al hospital, pude ver cómo funcionaba y lo que hacían las enfermeras. También vi partos y cesáreas [...] se me hacían 
padres [...]. Iba al hospital para que me agradara más la carrera". Estas acciones fortalecieron la illusio de Adelita y le permitieron incorporar disposiciones afines a la carrera de enfermería. Al mismo tiempo, su profesor se convirtió en modelo a seguir y en un capital social que le facilitó el ingreso a un hospital, lo que puede considerarse como capital cultural para la carrera de enfermería. De esta manera, Adelita consolidó su hexis a través de la incorporación temprana de disposiciones y los capitales cultural y social, afines al habitus profesional de la enfermería y al habitus de ingreso a la universidad, lo que a su vez reforzó su illusio de ingresar a esta carrera.

Los casos de Adelita y Mary muestran que existen estudiantes que desde etapas muy tempranas generan expectativas e incorporan disposiciones que refuerzan su interés en una carrera. Estas acciones prácticamente se interrelacionan con la elección de carrera y gradualmente fortalecen la illusio y la hexis del aspirante, es decir, sus intereses, expectativas y disposiciones. Esto les permitió identificar tempranamente las reglas explícitas e implícitas del campo universitario y de la carrera y emprender acciones prácticas encaminadas a responder adecuadamente a ellas; además, las llevó a adquirir capitales sociales y culturales que en conjunto contribuyeron a mantener su aspiración y a establecer estrategias para ingresar, como mejorar sus disposiciones escolares desde la preparatoria, y a superar las dificultades que enfrentaron, como ser rechazadas en su primer intento.

\section{Ruta 2: elección temprana con valoración de posibilidades}

En cuatro estudiantes se detectaron momentos de reflexión previos a la elección de carrea, reflexiones que generalmente iban enfocadas a las dificultades que enfrentarian para responder a las demandas de la carrera de interés y sus posibilidades de ingreso a la misma.

1 Mexicanismo por "buenos".
Los estudiantes en esta situación tenían dudas acerca de si su elección era la adecuada. Estas fueron resueltas de diferente manera. Raquel y Betty, ambas estudiantes de Enfermería, manifestaron que, además de esta carrera, mantenían interés por una segunda carrera; sin embargo, durante la secundaria y la preparatoria, respectivamente, generaron la creencia de que la otra carrera presentaba mayores exigencias de ingreso y permanencia, que creyeron no poder satisfacer. Ante ello, prefirieron hacer trámites a una carrera que, según creían, presentaría menos exigencias.

En las decisiones tomadas por Betty y Raquel se nota un ajuste en su ruta de ingreso, de manera que definieron su elección en función de su creencia de poder o no responder adecuadamente a las reglas del campo, aun antes de haber intentado ingresar. En ambos casos las aspirantes consideraron que la opción que eligieron también les gustaba previamente, es decir, descartaron una en función de sus creencias.

Susana también mostró dudas durante su elección y manifestó su temor a no ingresar, por lo que valoró otra carrera: "Ya en el momento de decidirme me daba miedo no quedar en listas y vi otras carreras, estaba entre [ser] químico farmacobiólogo, que también se me hacía interesante, y Medicina". La valoración de Susana permite identificar que la elección puede ser una etapa de incertidumbre, en la que el estudiante no cuenta con los elementos suficientes para para elegir una carrera. Pero ella tuvo un elemento con el que no contaron Raquel y Betty, pues se informó sobre ambas carreras gracias a una tarea durante la preparatoria, acción que reforzó su interés por la medicina y le ayudó a descartar la carrera que tenía como segunda opción. Al revisar las materias de la carrera de Medicina, Susana entró en contacto con el campo universitario, lo que le permitió reforzar su illusio al respecto y la llevó a mantener su elección. Además, con sus acciones prácticas Susana reconoció de manera implícita su capacidad para cumplir con las reglas del campo, es decir, con las exigencias de la carrera. 
Otro de los alumnos, Gonzalo, comenzó a interesarse por una carrera universitaria cuando estudiaba la secundaria, gracias a que uno de sus profesores era agrónomo de profesión y le inculcó el interés por el cultivo de plantas: "desde que entré a la secundaria, me llamó la atención que un profe cultivaba en lugares chiquitos, sacaba varias cosas que no había en las fruterías [...] yo iba con él y sí las tenía"; sin embargo, para estudiar Agronomía Gonzalo debía mudarse a la ciudad de Guadalajara, ante lo cual no manifestó disposiciones ni expectativas lo suficientemente consolidadas como para hacerlo. Al preguntarle por qué no se mudó, respondió: "En sí [el motivo fue] la distancia, porque estar hasta allá [...] no me llama la atención estar fuera de mi casa. No le hace que estuviera yendo y viniendo, pero estar en casa, con los amigos divirtiéndose. Porque si estuviera en otro lado como en Guadalajara o Lagos nomas estaría dentro de la casa sin distraerme".

Las valoraciones que realizó Gonzalo se enfocaron en los cambios que debía realizar para estudiar la carrera de Agronomía, vistos como distanciamiento de su casa y amigos; además, debido a que en ocasiones mencionó que tuvo la necesidad de pedir a su patrón un préstamo para pagar su matrícula, se asume que también evaluó las exigencias económicas que implicaba el cambio de ciudad. Ante esta situación, Gonzalo valoró las carreras que ofrecía el cualtos para estudiar una profesión que satisficiera su interés por el cultivo de plantas y al mismo tiempo permanecer en su casa y optó por hacer trámites para entrar a Sistemas Pecuarios.

\section{Ruta 3: elección tardía y poco informada}

Esta ruta se caracterizó por que los estudiantes eligieron su carrera poco antes de hacer trámites y previo a ello no habían pensado seriamente en ingresar a la universidad. En esta situación se encuentran José, Luis y Gerardo, quienes manifestaron interés en un área de conocimientos, se informaron someramente sobre las carreras relacionadas con dicha área y fueron descartando opciones, hasta elegir una. Un ejemplo de este proceso es el segui- do por José, quien decidió ingresar a la universidad para mantener una beca que obtuvo por ser instructor comunitario, la cual estipulaba como requisito mantenerse estudiando. Cuando José terminó la preparatoria, se vio en la necesidad decidir qué estudiar, valoró sus posibilidades y eligió como mejor opción una carrera universitaria, en vez de cursos o diplomados: "entonces dije: bueno, pues [Sistemas Pecuarios] es una buena opción". Como le gustaban el rancho, los animales y la producción agropecuaria, José comparó las carreras de Veterinaria, Agroindustrias y Sistemas Pecuarios y consideró que esta última respondía más a sus intereses. Para ello se apoyó en amigos que estudiaron esta carrera, quienes lo orientaron y advirtieron que no era una carrera fácil.

De los estudiantes que manifestaron una elección tardía, Luis y José se caracterizan por un fuerte interés en las labores agropecuarias y por ser cercanos a ellas, ya que colaboran de manera regular en los ranchos de sus padres. Esta cercanía con las actividades del rancho pudo orientar su elección de carrera, de manera que, aunque pareciera una decisión apresurada, ellos ya tenían un conocimiento empírico de la profesión; además, consultaron con egresados las características de la carrera de Sistemas Pecuarios. Con estas acciones lograron un alto grado de afinidad entre sus expectativas y disposiciones profesionales y lo que ofrece la carrera que eligieron.

Un caso en el que se muestran disposiciones y expectativas muy difusas y poco relacionadas con la carrera a la que el estudiante ingresó es el de Gerardo, quien tenía como opciones ser policía, estudiar Ingeniería Mecánica o Sistemas Pecuarios, aunque no mostró una valoración previa de ninguna carrera. "Al principio no estaba muy seguro, quería estudiar ingeniero mecánico [...] pero dije como que me gustaría aprender más cosas [...]. Siempre me ha gustado el rancho, la producción de animales". A pesar de lo anterior, mostraba un mayor interés en ser policía federal, "porque se gana mucho dinero", pero su mamá se opuso a ello e influyó en que hiciera trámites para la universidad. Además, duran- 
te las entrevistas no mencionó que hubiera hecho alguna valoración de la carrera que estudiaría, sino que siguió consejos poco sustentados de personas alejadas de la profesión: "ella me decía que como me visto así vaquero, pues pa' que traigas tus botas".

La falta de interés que muestra Gerardo para informarse adecuadamente sobre la carrera que estudia y el hecho de que su mamá lo presionó para realizar trámites reflejan bajas expectativas con la carrera y disposiciones poco afines al campo universitario. Este caso se configura de manera diferente al resto y destaca, por manifestar directamente que no quería seguir estudiando. Por lo que respecta a los casos de Luis y José, aunque tomaron su decisión en poco tiempo, lograron obtener información suficiente como para elegir una carrera afín a sus intereses y conociendo las exigencias de la misma.

\section{Ruta 4: cambio de aspiración}

Dos estudiantes establecieron el cambio de aspiración como estrategia de ingreso al campo universitario. Ellos se vieron obligados a elegir una carrera distinta a la que les interesaba originalmente. Aunque este cambio se generó por motivos distintos, refleja que fueron capaces de identificar sus limitantes y establecer acciones prácticas para ingresar a una carrera universitaria. Pedro eligió Enfermería después de que no fue admitido a Medicina. Ante este acontecimiento, valoró sus posibilidades y estableció un ajuste: el cambio de aspiración, lo que manifiesta de la siguiente manera: "cuando no entré a Medicina dije: para qué perder el tiempo [...] si quedé a veinte puntos, creo que voy a quedar igual. Y dije pues a Enfermería". Esta reflexión va de la mano con la búsqueda de argumentos que permitieron a Pedro resignificar su ingreso a la universidad: "creo que Enfermería es mejor porque llevas menos responsabilidades, o sea, el responsable es el doctor". De esta manera comenzó a ver las ventajas que le ofrecía la carrera de Enfermería, lo que cambió su illusio, pues empezó a identificar y generar expectativas positivas hacia la nueva carrera.
El cambio de aspiración de Pedro se entiende como un ajuste encaminado a responder a las reglas del campo universitario, pues, al estimar que su puntaje en el proceso de admisión a Medicina no le permitiría ingresar, optó por aspirar a otra carrera, a la cual, según su valoración, sí podría acceder. La ruta seguida por Pedro hace evidente que los aspirantes son capaces de valorar sus capitales culturales, económicos y sociales, estimar sus posibilidades y, en función de ello, tomar decisiones que les permitan ingresar a la universidad. Aun a pesar de que la elección de Pedro surgió de restricciones que el propio campo universitario le impuso, él tuvo la capacidad de generar nuevas expectativas y disposiciones que le permitieran valorar positivamente otra carrera y con ello ingresar a la universidad.

En el caso de Salvador intervinieron valoraciones y aspiraciones de tipo personal, familiar y escolar que desembocaron en el cambio de carrera. Él había ingresado a su carrera de interés, que era Ingeniería de Sistemas, pero mostró insatisfacción respecto a la calidad de la institución donde estudiaba, por lo que desertó y buscó el apoyo de sus padres para estudiar la misma carrera en Guadalajara, situación que rechazaron. Ante este escenario, Salvador decidió ingresar a una carrera que le consiguiera el apoyo de sus padres para salir de su pueblo, y esto lo encontró en Medicina: "Yo iba estudiar ingenierías en León, me quedaba cerquita de donde vivo, pero no me llenaba la escuela, ni la carrera. Entonces busqué otra opción para que mi mamá me apoyara, [y así] fue como decidí estudiar Medicina [...]. Cuando les dije a mis papás de Medicina me dijeron que sí. Ellos me apoyaban si quería esperarme [para entrar a la UdeG] o me pagaban una particular [...] en Guadalajara".

Estos apoyos diferenciados muestran que los padres de Salvador estuvieron dispuestos a subvencionar Medicina debido a que valoran más los capitales que ofrece esta carrera. Así, indujeron en él un cambio de illusio. Además, se aprecia que sus padres se convirtieron en un capital social que le permitió 
cambiarse de ciudad. Ante el cambio de aspiración, Salvador manifestó interés por una carrera de prestigio que representara un reto y estos elementos se incorporaron a su illusio, mientras que las aspiraciones y deseos de conocimientos propios de la carrera se fueron incorporando al irla cursando: "Ouería una carrera que valiera la pena, no cualquiera, y Medicina se me hace bien: es como un reto estudiarla, algo que no cualquiera puede hacer, y por eso fue que decidí echarme el compromiso de Medicina [...] le vas agarrando cariño, ya me emociona ir a ver el cadáver y empezar a ver qué tienes o qué sientes".

La capacidad de valorar sus opciones, estimar sus posibilidades y elegir entre ellas permite apreciar cierta habilidad en Salvador para comprender las reglas del campo universitario y establecer acciones que maximicen sus resultados a partir de sus capitales y disposiciones incorporadas, en este caso, ingresar a la carrera de Medicina en el cualtos.

\section{Variante: volver a hacer trámites}

Un momento clave durante el proceso de ingreso a la universidad, que permite apreciar la fuerza de las reglas que impone el campo universitario y contrastarlas con las acciones prácticas del estudiante, sucedió cuando algunos de los entrevistados recibieron la notificación de que no fueron admitidos en la carrera para la que hicieron trámites, durante un proceso de selección previo. Fue en el momento en el que supieron su puntaje en el proceso de admisión y lo compararon con el requerido para ingresar a la carrera deseada cuando tuvieron elementos certeros para valorar sus capitales culturales y contrastarlos con los que el cualtos, entendido como campo, exige para sus carreras.

Una vez que cada alumno tuvo dicha información, estableció una estrategia de ajuste que varió en cada sujeto a partir de sus expectativas, disposiciones incorporadas y capitales. De esta manera, siete de los doce entrevistados presentaron la variante volver a hacer trámites, lo que los llevó por procesos reflexivos y de toma de decisiones diferentes: Adelita, Mary, Susana y Andrea no ingresaron durante su primer intento e hicieron trámites para la misma carrera y en la misma institución; Rebeca hizo trámites para la misma carrera en diferente institución; Pedro hizo trámites para una carrera distinta en la misma institución; y Betty ingresó a la carrera deseada después de varios intentos en la misma institución. Aunque todos tomaron caminos y decisiones diferentes, sus acciones desembocaron en el ingreso al campo universitario.

En el caso de volver a hacer trámites para la misma carrera en el mismo campo, la única opción que tiene el aspirante para mejorar sus posibilidades de ingreso es elevar el puntaje del examen de admisión, pues el promedio de preparatoria resulta de la suma de pequeñas acciones por un periodo largo y, una vez terminada la preparatoria, es imposible su modificación; por tanto, constituye un reflejo de sus disposiciones incorporadas.

Otro ajuste consistió en buscar ingresar a la misma carrea en un campo con reglas de ingreso menos exigentes. Este fue el caso de Raquel, quien antes de entrar al cualtos hizo trámites para la carrera de enfermería en el Centro Universitario de Ciencias de la Salud (CUCS), un centro metropolitano de la misma UdeG que exige un mayor puntaje de ingreso. De esta manera, se puede considerar al cualtos como un campo con menos prestigio que el CUCS, pero que básicamente ofrece los mismos capitales: estudiar una carrera universitaria específica.

En cuanto al cambio de carrera, aunque ya se explicó líneas arriba como una ruta específica, vale señalar que al analizarla a partir de la variante de volver a hacer trámites se hace evidente una profunda modificación en las expectativas del estudiante, es decir, de su habitus de ingreso a la universidad. Respecto al ingreso a la universidad y su relación con la elección de carrera, se puede afirmar que los estudiantes emprenden acciones prácticas con el fin de ser admitidos, las cuales están influi- 
das por sus disposiciones y capitales, y en diferentes momentos se pueden presentar ajustes a partir de las exigencias del campo universitario. Estos ajustes muchas veces demuestran la capacidad del estudiante para percibir las reglas del campo universitario y valorar sus capitales y disposiciones, lo que le permite establecer estrategias efectivas de ingreso. En la Figura 1 se muestran, de manera general, los elementos y valoraciones que componen las rutas de ingreso seguidas por los estudiantes, las cuales inician con el interés por una carrea específica, pero pueden desarrollarse de maneras distintas, esto a partir del habitus de ingreso a la universidad, los capitales y la interacción de cada estudiante con el campo universitario.

El análisis de los datos muestra que la elección de carrera se genera en la subjetividad del aspirante, pues surge del conocimiento y comprensión que tiene sobre las reglas del campo universitario. In- gresar a la universidad se puede entender como el momento en que el estudiante se abre las puertas de la universidad a través de sus acciones prácticas. Proceso que, como pudo apreciarse, implica una interacción dialéctica entre el campo universitario y el aspirante, a través de la cual este último establece sus propias estrategias sustentadas en sus capitales y guiadas por su habitus, para cumplir con las reglas que el campo establece.

\section{Discusión}

El propósito del presente estudio fue comprender el proceso de ingreso a la universidad vivido por estudiantes del Centro Universitario de Los Altos, de la Universidad de Guadalajara. Para ello se apoyó en los conceptos teóricos de campo, capital y habitus desarrollados por Bourdieu, que permitieron analizar el problema desde una perspectiva dialéctica, en la que se entiende al cualtos como una estructura

Figura 1. Rutas de ingreso a la universidad

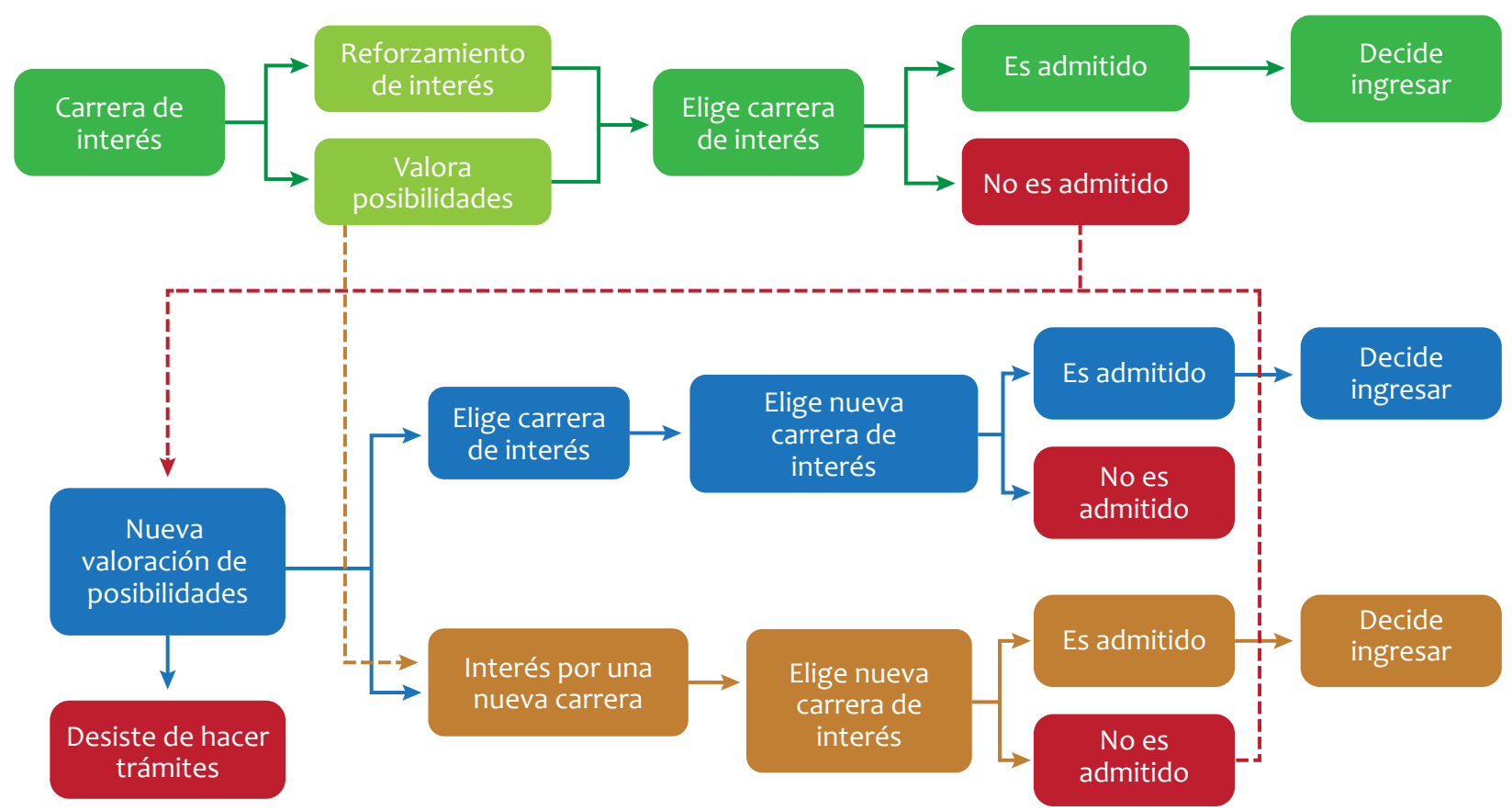

Fuente: elaboración propia. 
social que establece reglas y a los estudiantes como personas activas que, desde su propia subjetividad, e impulsados por su habitus, ponen en juego sus distintos capitales (cultural, social, económico...), con el fin de ingresar a la universidad.

Desde esta perspectiva, el ingreso a la universidad se entiende como un proceso que comienza con el interés por estudiar una carrera y culmina con la admisión. Durante este proceso el entonces aspirante comienza, de manera incipiente, a generar expectativas y a incorporar disposiciones que lo impulsan a desplegar estrategias de ingreso a una carrera. De manera similar a lo encontrado por Grodsky y Riegle (2010), se identificó que el interés por ingresar a la universidad puede surgir a edades tempranas y que, cuando esto sucede, los aspirantes tienen mayores probabilidades de cumplir sus metas, debido a que son capaces de establecer estrategias más claras y efectivas, en comparación con quienes lo hicieron de manera tardía.

Existen estudios que se han enfocado en cómo influye la escuela de procedencia en la incorporación de disposiciones que impulsen a los estudiantes a ingresar a la universidad, lo que se ha denominado habitus institucional (González, 2016; Langenkamp y Shifrer, 2011; Reay et al., 2001). A diferencia de dichas investigaciones, en este estudio fueron pocas las menciones a mecanismos institucionales de promoción de estudios superiores que hayan influido en la intención de ingresar a la universidad. En contrapartida, fue más común que los estudiantes se refirieran a mecanismos no formales, como pláticas y consejos de profesores.

Respecto a los ajustes que los estudiantes realizaron para ingresar a la universidad, destaca la manera en que fueron haciendo una elección razonada. En este sentido, y de manera similar a lo encontrado por Piñero (2015), algunos estudiantes descartaron carreras que les interesaban, por considerar que no podrían cumplir con ciertas exigencias académicas, económicas y/o de ingreso. En cuanto a las desigualdades en el ingreso y elección de carrera provocadas por las diferencias de género, los y las estudiantes entrevistadas no mencionaron alguna dificultad atribuible a este factor, lo que difiere de lo encontrado en otras investigaciones (García et al., 2012; Navarro y Casero, 2012), aunque es pertinente aclarar que el presente estudio no tuvo como eje central esta cuestión.

Si bien el concepto de habitus -ya sea en su forma genérica (Lehmann, 2013) o en sus formas específicas de habitus institucional (González, 2016; Langenkamp y Shifrer, 2011) o habitus de ingreso a la universidad (Grodsky y Riegle, 2010)- ha permitido comprender que existen disposiciones que impulsan a los estudiantes a ingresar a la universidad, dichos trabajos se han enfocado principalmente en las disposiciones de carácter escolar y han dejado de lado las profesionales. Estas últimas han permitido a la presente investigación hacer evidente que el aspirante busca ingresar a la universidad no solo para continuar sus estudios, sino que en realidad pretende ingresar a un acampo profesional para el cual es requisito tener un título universitario.

El interés por una profesión y el modelo como elemento importante para desarrollar el deseo de ingresar a la universidad permitieron detectar y comprender el surgimiento del habitus de ingreso a la universidad en alumnos provenientes de contextos tanto alejados como cercanos al campo universitario. También, a partir de ello, se pudo esclarecer cómo los capitales sociales de los jóvenes pueden, desde edades muy tempranas, contribuir o no a la incorporación del habitus de ingreso a la universidad y, por tanto, al inicio de acciones prácticas que de manera gradual, pero constante, acercan a los jóvenes al campo universitario. Esto complementa los hallazgos de las investigaciones que hacen evidente que el ingreso a la universidad está relacionado con los capitales y habitus de los estudiantes, pero no profundizan en cómo surge el interés por ingresar a la universidad (Grodsky y Riegle, 2010). 
Por último, y de acuerdo con lo planteado por Edgerton y Roberts (2014), la presente investigación buscó utilizar de manera integral los conceptos de habitus, capital y campo, como elementos complementarios. Esto permitió identificar diferentes rutas de ingreso a la universidad y describirlas como acciones prácticas encaminadas a responder a las reglas que impone el campo universitario y sus carreras, las cuales fueron impulsadas por el habitus de ingreso a la universidad y sustentadas en los capitales de cada estudiante.

\section{Conclusiones}

Los hallazgos del presente trabajo permiten comprender la importancia de la incorporación del habitus de ingreso a la universidad a edades tempranas, que ocurre a través de los contactos que tiene el aspirante con modelos capaces de generar expectativas y disposiciones tanto escolares como profesionales. Generar tales expectativas y disposiciones se dificulta en los casos de estudiantes provenientes de familias en contextos desfavorecidos y alejados del campo universitario. En estos casos, los niveles de estudio previos pueden fungir no solamente como espacios de transmisión de conocimientos, sino como uno de los pocos lugares en los que los alumnos, independientemente de su origen social, pueden convivir con profesionistas, que, como modelos, tienen el potencial de generar en los estudiantes expectativas y disposiciones de tipo profesional.

Por otro lado, si se entiende la universidad como campo o estructura social que establece reglas de ingreso y permanencia a las cuales el estudiante debe responder, se hace evidente la importancia de que los jóvenes las conozcan lo antes posible, con la intención de incrementar su capacidad de agencia y estén en condiciones de mejorar sus posibilidades de ingreso. Un ejemplo de estas reglas es el promedio de la preparatoria, un capital cultural que se construye a lo largo de un proceso que, una vez concluido, no es posible modificar. De esta manera, concientizar a los estudiantes de primer semestre de preparatoria sobre la importancia de un promedio alto para incrementar sus posibilidades de ingreso a la universidad podría impulsar en ellos acciones encaminadas a mantener altas notas, lo que a su vez consolidaría su habitus de ingreso a la universidad.

Atendiendo a lo anterior y reconociendo que existen espacios en los que es difícil intervenir, por ejemplo, en las condiciones socioculturales de los estudiantes, cobra relevancia la preparatoria como espacio institucional que promueva políticas especialmente diseñadas para inculcar en los estudiantes el habitus de ingreso a la universidad. Por ejemplo: generar mecanismos a través de los cuales el docente se muestre no solo como profesor sino como profesionista, con el fin de que funja como modelo y genere interacciones similares a las experiencias de Adelita y Gonzalo, cuando sus profesores en horarios extraescolares los acercaron a actividades profesionales de las carreras a las que aspiraban ingresar.

Para finalizar, es importante mencionar que el estudio se realizó en un centro universitario con características específicas, entre las que destaca su carácter público, regional y multitemático; por ello, no es posible generalizar los hallazgos a toda la población universitaria, pues, aunque los casos fueron analizados en profundidad, solo fueron doce y todos en un escenario específico; sin embargo, la investigación contribuye a comprender el fenómeno en este contexto particular y sienta las bases para elaborar nuevos estudios de tipo confirmatorio. 


\section{Referencias}

Bernardi, F. y Cebolla-Boado, H. (2014). Previous school results and social background: Compensation and imperfect information in educational transitions. European Sociological Review, 30(2), 207-217. https://doi. org/10.1093/esr/jcto29

Bourdieu, P. (1990). Sociología y cultura. Conaculta-Grijalbo.

Bourdieu. P (2002). Campo de poder, campo intelectual. Itinerario de un concepto. Montressor.

Bourdieu, P. (2008). Homo academicus. Siglo XXI.

Bourdieu, P. y Passeron, J.C. (2003). Los herederos. Siglo XXI.

Bourdieu, P. y Wacquant, L. (1995). Respuestas por una antropología reflexiva. Grijalbo.

De Garay, A. (2004). Integración de los jóvenes en el sistema universitario. Prácticas sociales, académicas y de consumo cultural. Pomares.

Edgerton, J. D. y Roberts, L. W. (2014). Cultural capital or habitus? Bourdieu and beyond in the explanation of enduring educational inequality. Theory and Research in Education, 12(2), 193-220. https://doi. org/10.1177/1477878514530231

Farías, I. (2010). Adieu à Bourdieu? Asimetrías, límites y paradojas en la noción de habitus. Convergencia, 54 (septiembre), 11-34. http://www.scielo.org.mx/pdf/conver/v17n54/v17n54a1.pdf

García, J., Ávila, D., Vargas, M. y Hernández, C. (2015). Acerca de la feminización de profesiones. Caso: la docencia en preescolar en la Ciudad de México. Revista de Estudios de Género: La Ventana, 5(42), 129-151. http://www. scielo.org.mx/pdf/laven/v5n42/1405-9436-laven-5-42-00129.pdf

García, J., Hernández, C. I. y Moreno, D. (2012). Campos profesionales sexualizados. La era femenina en educación. Revista Entre Maestr@s, 16/17(59/60), 78-88. http://editorial.upnvirtual.edu.mx/index.php/entremaestrs/10-revista-entre-maestr-s/382-numero-59-60

González, Á. (2014). Aspirar a una universidad selectiva y concretar las aspiraciones: factores determinantes. Calidad en la Educación, 40 (julio), 235-267. https://doi.org/10.4067/So718-45652014000100008

González, Á. (2016). Cultura institucional de promoción de estudios universitarios y proceso de elección de estudios. Estudios Pedagógicos, XLI/(3), 171-189. http://dx.doi.org/10.4067/S0718-07052016000400009

Grodsky, E. y Riegle-Crumb, C. (2010). Those who choose and those who don't: Social background and college orientation. The annals of the American Academy of Political and Social Science, 627(1), 14-35. https://doi. org/10.1177/0002716209348732

Guerra, M. I. (2009). Trayectorias formativas y laborales de jóvenes de sectores populares. Anuies. 
Guzmán, C. (2012). Quedar afuera: experiencias y vivencias de los jóvenes que no logran ingresar a la universidad. Cultura y Representaciones Sociales, 6(12), 131-164. http://www.culturayrs.unam.mx/index.php/CRS/ article/view/418

Guzmán, C. y Saucedo, C. (2005). Aproximaciones y elaboraciones conceptuales sobre los alumnos. Aportes de diversos países. En P. Ducoing (ed), Sujetos, actores y procesos de formación. T. II: La investigación sobre alumnos en México: recuento de una década (1992-2002) (pp. 649-658). Comie.

Hegna, K. (2014). Changing educational aspirations in the choice of and transition to post-compulsory schooling-a three-wave longitudinal study of Oslo youth. Journal of Youth Studies, 17(5), 592-613. https://doi.org $/ 10.1080 / 13676261.2013 .853870$

Langenkamp, A. G. y Shifrer, D. (2011). Developing a college-going habitus: How families and schools influence first generation students. [Paper] Annual Meeting of the American Sociological Association Annual Meeting, Caesar's Palace, 1-35. http://citation.allacademic.com/meta/p507294_index.html

Lehmann, W. (2014). Habitus transformation and hidden injuries: Successful working-class university students. Sociology of Education, 87(1), 1-15. https://doi.org/10.1177/0038040713498777

Macías, G. G., Caldera, J. F. y lñiguez, M. de J. (2011). Evolución de la matrícula femenina en la educación superior de la región Altos Sur de Jalisco: el caso del Centro Universitario de los Altos. Revista de Educacióny Desarro1lo, 18 (julio), 1-26. https://www.cucs.udg.mx/revistas/edu_desarrollo/anteriores/18/018_Macias.pdf

Navarro, C. y Casero, A. (2012). Análisis de las diferencias de género en la elección de estudios universitarios. Estudios sobre Educación, 22, 115-132. https://revistas.unav.edu/index.php/estudios-sobre-educacion/article/ view/2075/1940

Miller, D. (2015). Exploración del vínculo entre trayectorias escolares y marcos normativos en la Universidad Autónoma. Revista Iberoamericana de Educación Superior, 6(17), 21-37. https://doi.org/10.1016/j.rides.2015.10.001

Mota, M. (2013). El habitus internético escolar de estudiantes de la Universidad Autónoma de Aguascalientes. [Tesis doctoral en Educación] Instituto Superior Tecnológico y de Estudios Superiores de Occidente, Guadalajara. https://rei.iteso.mx/handle/11117/1203

Ochoa M., R. (2000). El College Board. Efectos sociales y académicos de los criterios de selección de alumnos en una universidad pública. Universidad de Guadalajara.

Piñero, M. S. L. (2015). Factores asociados a la selección de carrera: una aproximación desde la Teoría de la Acción Racional. CPU-e. Revista de Investigación Educativa, 20, 72-99. https://doi.org/10.25009/cpue.voi20.1288

Pérez, F. y Villarruel, M. (2016). Desigualdad en el acceso educativo en México: un estudio con sujetos egresados de un bachillerato tecnológico de alto desempeño. Revista Electrónic@ Educare,20(3), 1-22. https://doi. org/10.15359/ree.20-3.21

Reay, D., David, M. y Ball, S. (2001). Making a difference?: Institutional habituses and higher education choice. Sociological Research Online, 5(4), 1-12. https://doi.org/10.5153/sro.548 
Rodríguez, S., Fita, E. y Torrado, M. (2004). El rendimiento académico en la transición secundaria-universidad. Revista de Educación, 334, 391-414. http://www.revistaeducacion.educacion.es/re334/re334_22.pdf

Rodríguez, M. C., Peña, J. V. y García, O. (2016). Estudio cualitativo de las diferencias de género en la elección de opciones académicas en los estudiantes del bachillerato científico-técnico. Teoría de la Educación, 28(1), 189-207. https://doi.org/10.14201/teoredu2016281189207

Silva, M. y Rodríguez, A. (2012). El primer año universitario entre jóvenes provenientes de sectores de pobreza: un asunto de equidad. Anuies.

Subero, D. y Esteban-Guitart, M. (2017). La mejora de las expectativas educativas en un centro abierto de Barcelona. Un proyecto de orientación educativa para jóvenes en riesgo de exclusión social. Cultura y Educación, 29(2), 285-394. https://doi.org/10.1080/11356405.2017.1306989

Taylor, S. J. y Bogdan, R. (1996). Introducción a los métodos cualitativos de investigación. Paidós.

Velasco, D. (2000), Habitus, democracia y acción popular. La sociología de Pierre Bourdieu aplicada a un estudio de caso. Iteso.

Warren, C. (2001). Qualitative interviewing. En J. F. Gubrium y J. A. Holstein (eds.), Handbook of interview research (pp. 83-101). Sage. 